\title{
The Dispersion Process based on Organic-inorganic Composite Technology and its Application in Thermal Reflective Insulation Coatings
}

( 1 Materials and Metallurgical College, Northeastern University, Shenyang 110819, China)

( 2 State Key Laboratory of Metastable Materials Science and Technology, Yanshan University, Qinhuangdao 066004, China)

( 3 Hebei Provincial Laboratory for Dielectric and Electrolyte Materials, Northeastern University at Qinhuangdao, Qinhuangdao 066004, China)

( 4 Beijing National Laboratory for Molecular Sciences, Institute of Chemistry Chinese Academy of Sciences, Beijing 100190, China)

\section{Introduction}

The temperature will rise greatly on the surface of building, communication power equipment, oil storage tank and other objects through strong solar radiation, which may bring much inconvenience to human production and life. It is an urgent need to reduce the harm of heat radiation for building energy efficiency. A large number of studies have shown that painting thermal reflective insulation coating on the surface of building, instruments and oil storage tanks is an effective way to lower the surface temperature of the object. However, the current study on thermal reflective insulation coating is in the development stage[1].

Titanium dioxide is widely used in thermal reflective insulation coating as pigments and fillers due to its high refractive index and non-toxic and harmless chemical properties. However, it has a high surface energy, and is easy to generate agglomeration, after which the effect of thermal reflective insulation coating may be greatly reduced[2-3]. Therefore, it appears very important to proceed dispersion and modification of titanium dioxide filler. As Dandan Ye et al. mentioned in the overview, the surface treatment of thermal reflective insulation coating is used to improve the compatibility of the filler and the coating base material, which is the main development direction of thermal reflective insulation coatings.

The surface of titanium dioxide through organic surface modification will absorb or bond some organics or polymers; as a result, it can change the surface physical and chemical properties of titanium dioxide, reduce its surface energy, and improve the compatibility of titanium dioxide and organic media.

There are three methods for organic modification of titanium dioxide: coupling reagent, surface active agent and polymer encapsulation. Jianping Sun et al.[4] mentioned in the overview that, the modified titanium dioxide has relatively stable dispersion by using polymer encapsulation but its encapsulation procedure is rather complicated, compared to coupling reagent and surface active agent. In recent years, studies on surface
Abstract: With the photochemical activity of titanium dioxide, the surface polymer encapsulation is conducted to titanium dioxide under ultraviolet light initiation. It studies the influence of light source type, light exposure time and rate of charge on the product structure; the dispersion of titanium dioxide was compared before and after modification by using light absorption method; the modified titanium dioxide was prepared into thermal reflective insulation coating, and the difference of thermal barrier effect among uncoated, rock wool board and thermal reflective insulation coating was contrasted by self-made equipment.

Key words: Titanium dioxide; Modification; Dispersion; Heat reflection insulating mould coating

Published online: $30^{\text {th }}$ Nov, 2017 Corresponding author: Bingzhu Wang, E-mail:975128680@qq.com 
modification of titanium dioxide have been mainly focused on polymer encapsulation[5-6].

Photochemical activity of titanium dioxide was used in this paper. Under ultraviolet light initiation, the titanium dioxide surface generates free radicals to cause polymerization of acrylamide monomers on the surface of titanium dioxide. The generated poly-acrylamide polymer is coated on the surface of titanium dioxide particles. Compared with existing surface grafting and coating, it simplifies operation process and easier to make industrial extension. It's shown in Figure 1.

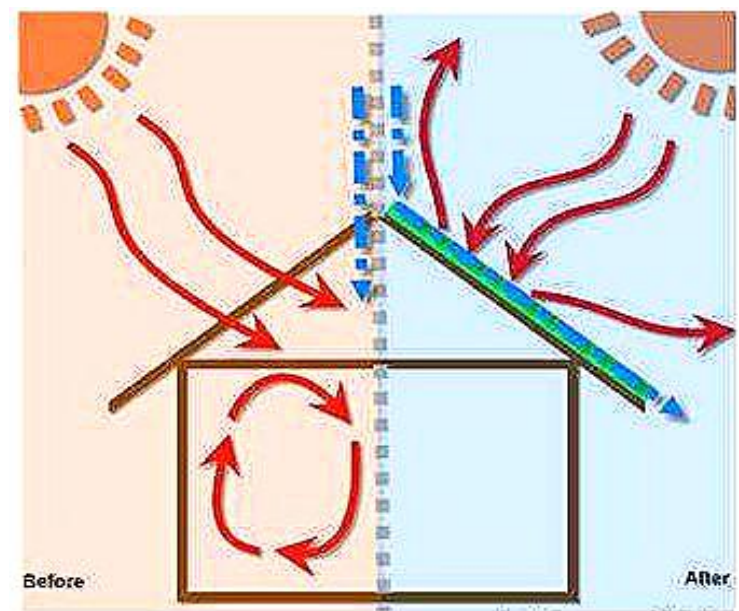

Figure 1 Principle of thermal insulation of thermal reflective insulation coating

\section{Experimental Material and Method}

\subsection{Experimental Material and Equipment}

Titanium dioxide, AR, Guangzhou Heqian Co., Ltd.; acrylamide, AR, Tianjin Kermel Chemical Reagent Co., Ltd.; absolute ethyl alcohol, AR, Tianjin Kaitong Chemical Reagent Co. Ltd.

Photochemical reaction instrument, LY-GHX-V, Shanghai Lanyi Industrial
Co., Ltd.; Fourier transform infrared spectrometer, FTIR-8400S, Beijing SHIMADZU Instrument Plant; thermogravimetry - differential thermal analyzer, HCT-2, Beijing Henven Scientific Instrument Factory; Centrifuge, HC-3018, Anhui Zonkia Scientific Instrument Co., Ltd.; electric air dry oven, 101-3AB, Tianjin Taisite Instrument Co., Ltd.; Ultrapure water machine, SP-780, Zhongshan Risheng Electrical Products Co., Ltd.

\subsection{Experimental Method}

Put a certain amount of $\mathrm{TiO}_{2}$ and deionized water in the quartz tube, stir evenly by magneton, weigh a certain amount of acrylamide to mix into the dissolution, inject nitrogen into the quartz tube, discharge the air, expose under ultraviolet light, take the sample out and move to the centrifugal tube, centrifuge in a centrifuge for $3 \mathrm{~min}$, pour the supernatant fluid out to leave surplus precipitation, wash the centrifuge with ethanol twice, and make it dry without air. The experimental operation flow is shown in Figure 2.

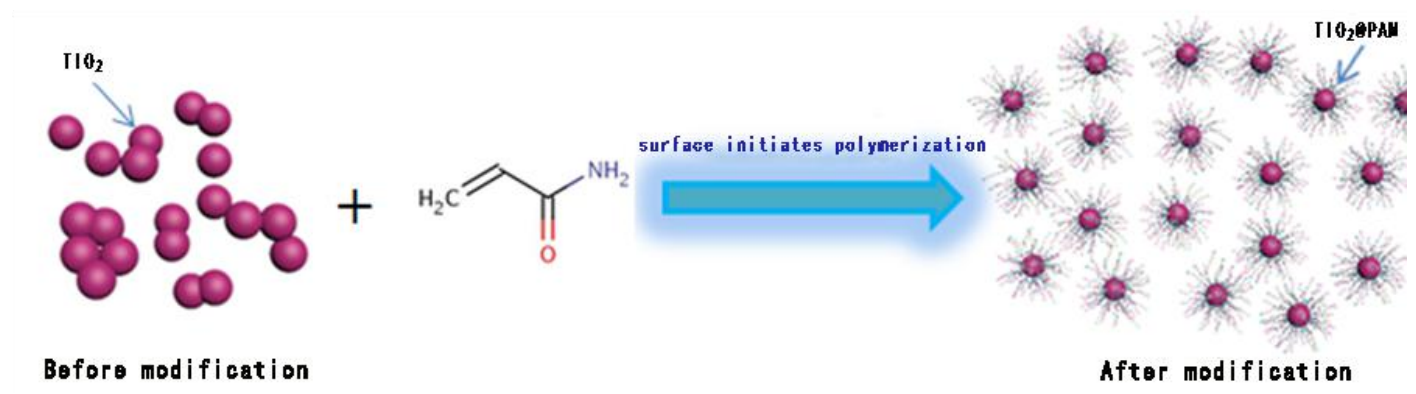

Figure 2 Schematic diagram of technical thinking

\section{Results and Discussions}

\subsection{Influence of $\mathrm{TiO}_{2}$ Dosage on Product}

\section{Dispersion}

In order to explore the solubility of acrylamide - titanium dioxide compound

in water, $0.01 \mathrm{~g}$ of samples were weighted 


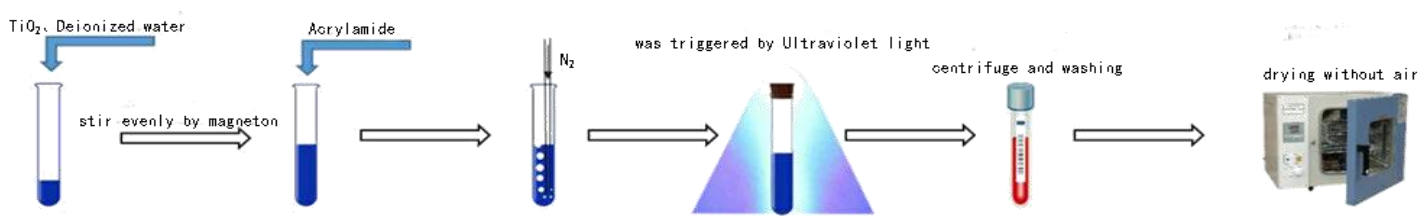

Figure 3 Flow chart of experimental operation

Table1 Solubility of product in water after $\mathrm{TiO}_{2}$ variable

\begin{tabular}{cc}
\hline $\mathrm{TiO}_{2}$ dosage/g & Solubility in water \\
\hline 0.1 & Partial solubility \\
\hline 0.075 & Partial solubility \\
\hline 0.05 & Complete solubility \\
\hline 0.025 & Complete solubility
\end{tabular}

It can be seen from the above table that the products are more soluble if the quality of titanium dioxide and acrylamide is lower. It is because the encapsulation rate of the polymer increases gradually with decrease of titanium dioxide content, and the products are more easily dispersed in aqueous solution. The thermal reflective insulation coating prepared is more uniform and stable, with better thermal reflection effect.

\subsection{Product Molecular Weight Analysis}

It can be seen from the Figure 4 that there is a peak at 18 to $20 \mathrm{~min}$, which is formed through flow of polyacrylamide, indicating that titanium dioxide has triggered polyacrylamide monomer into polymerization, to form polymer.

\subsection{Analysis on Product Thermal Stability}

Curve comparison of modified titanium dioxide $\left(\mathrm{TiO}_{2} @ P A M\right)$ and pure $\mathrm{TiO}_{2}$ thermal analysis is shown in Figure 4.
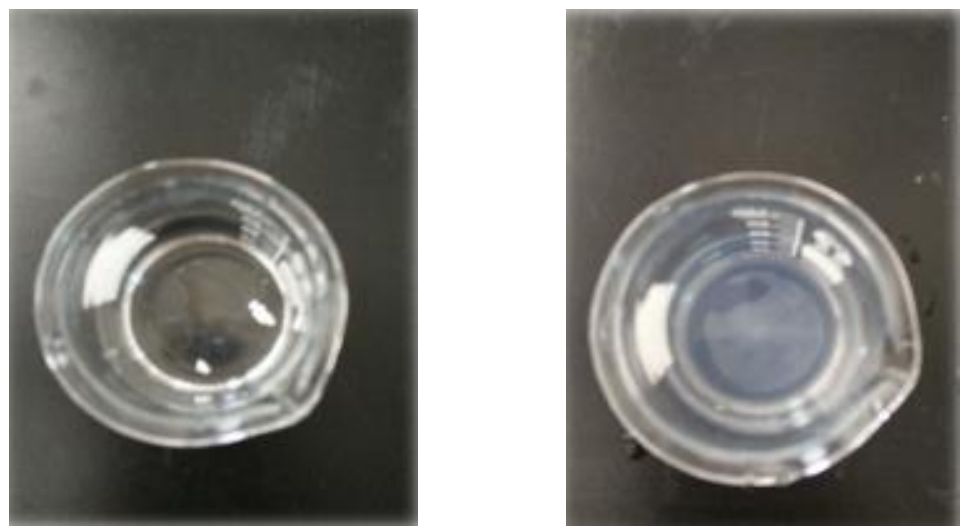

Figure 4 (a) Before dissolutionFigure 4(b) After dissolution

It can be seen from Figure 8 (a) and Figure 8 (b) that $\mathrm{TiO}_{2} @ P A M$ composite material has a significant exothermic peak between $300{ }^{\circ} \mathrm{C}$ to $700{ }^{\circ} \mathrm{C}$ compared to pure $\mathrm{TiO}_{2}$, indicating that the polyacrylamide polymer chain coated with $\mathrm{TiO}_{2}$ had oxygenolysis and released heat. The conclusion is consistent with that from high polymer liquid chromatography. 


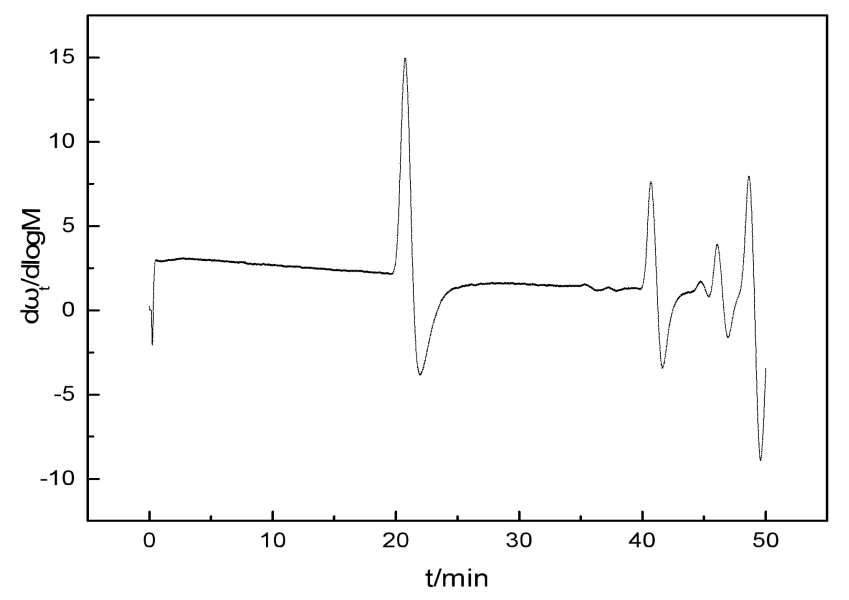

Figure 5 Gel chromatogram

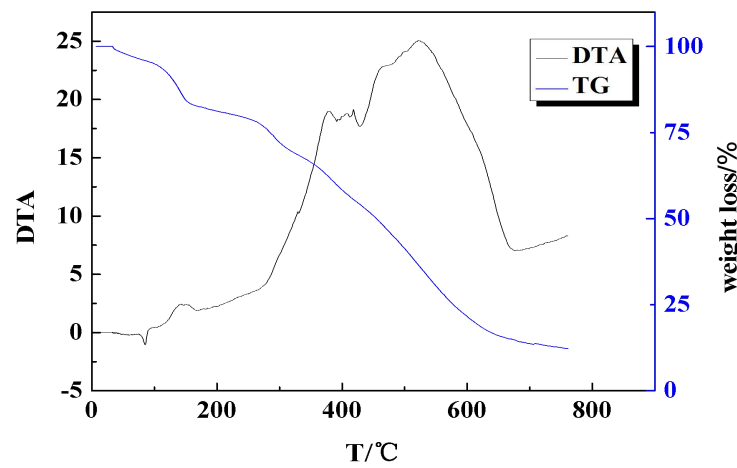

Figure 6 (a) ${\mathrm{PAM}-\mathrm{TiO}_{2}}$

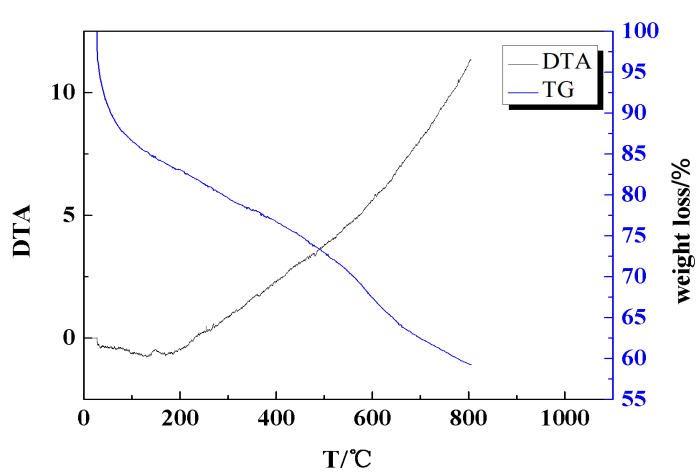

Figure $6(\mathrm{~b}) \mathrm{TiO}_{2}$

\subsection{Exploration of Product Dispersion}

Take $1 \mathrm{~g}$ of titanium dioxide to make coating modification, and the product is dispersed in $100 \mathrm{~mL}$ of deionized water. Another $1 \mathrm{~g}$ of titanium dioxide powder is dispersed in $100 \mathrm{~mL}$ of deionized water. The visible spectrophtometer is used to measure changes in the relationship between transmission ratio over time. The results are shown in Figure 7(a) and Figure 7(b).

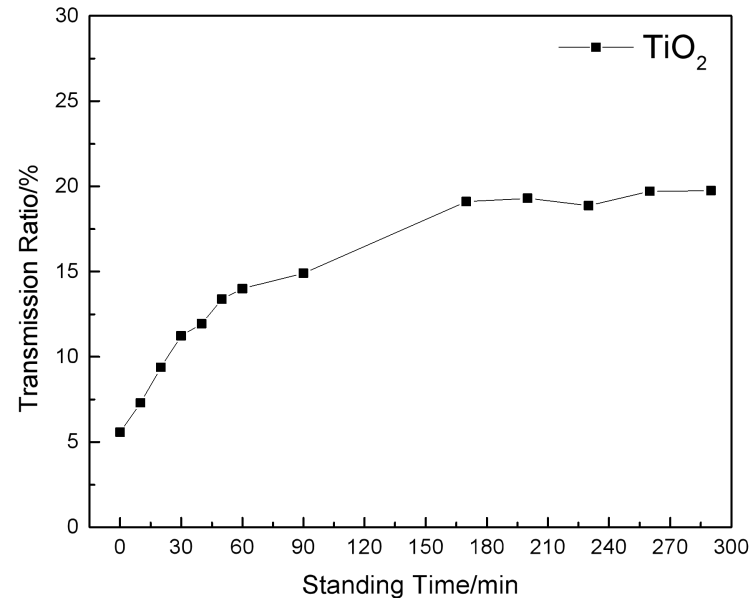

Figure 7 (a) Transmission ratio of unmodified $\mathrm{TiO}_{2}$ after dispersion

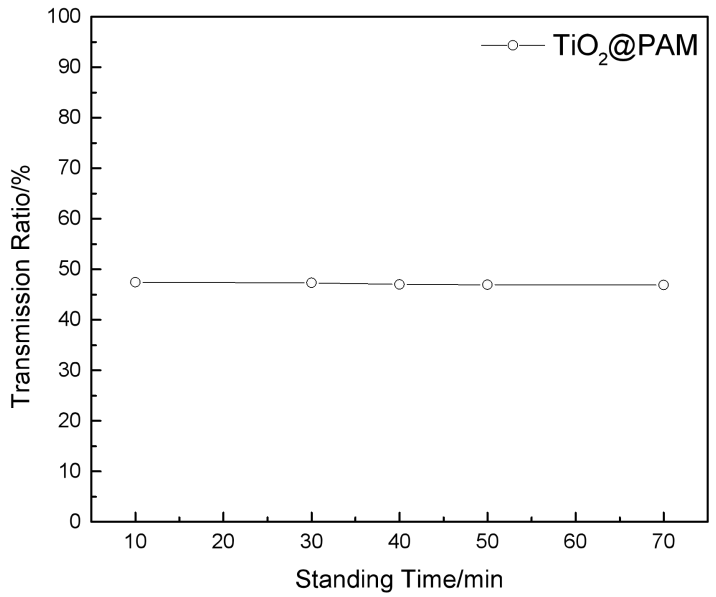

Figure 7(b) Transmission ratio of TiO ${ }_{2} @ P A M$ after dispersion
It can be seen from contract between Figure 10(a) and Figure 10(b) that the transmission ratio of solution changes over time and the change rate gradually decreases after the unmodified titanium dioxide is dispersed in deionized water, indicating that the titanium dioxide particles had sedimentation, with poor dispersion performance. The transmission ratio of $\mathrm{TiO}_{2}$ ( $\left.\mathrm{TiO}_{2} @ P A M\right)$ hardly changes over 
time, indicating that the formed system is rather stable, and the dispersion performance of titanium dioxide has been improved.

\subsection{Thermal Barrier Effect Test}

The modified titanium dioxide is prepared into thermal reflective insulation coating according to the research methods of Guozhong $\mathrm{Lu}^{[7]}$, thermal barrier effects are demonstrated by using devices shown in Figure 11, frame iron sheet is removed in three test areas, and polyurethane foams are applied to fill space, to avoid metal thermal affecting the experiment result. The insides of iron sheet in three test areas are designed into untreated sheet, sticking rock wool board, and coated with energy-saving thermal reflective insulation coating respectively. The iron box inside represents indoor environment, and electric heating lamp is used to heat the iron box inside. By examining the surface temperature of the outer surface of the iron box in three test areas, the thermal reflection insulation effect is compared in this paper ${ }^{[8-10]}$.

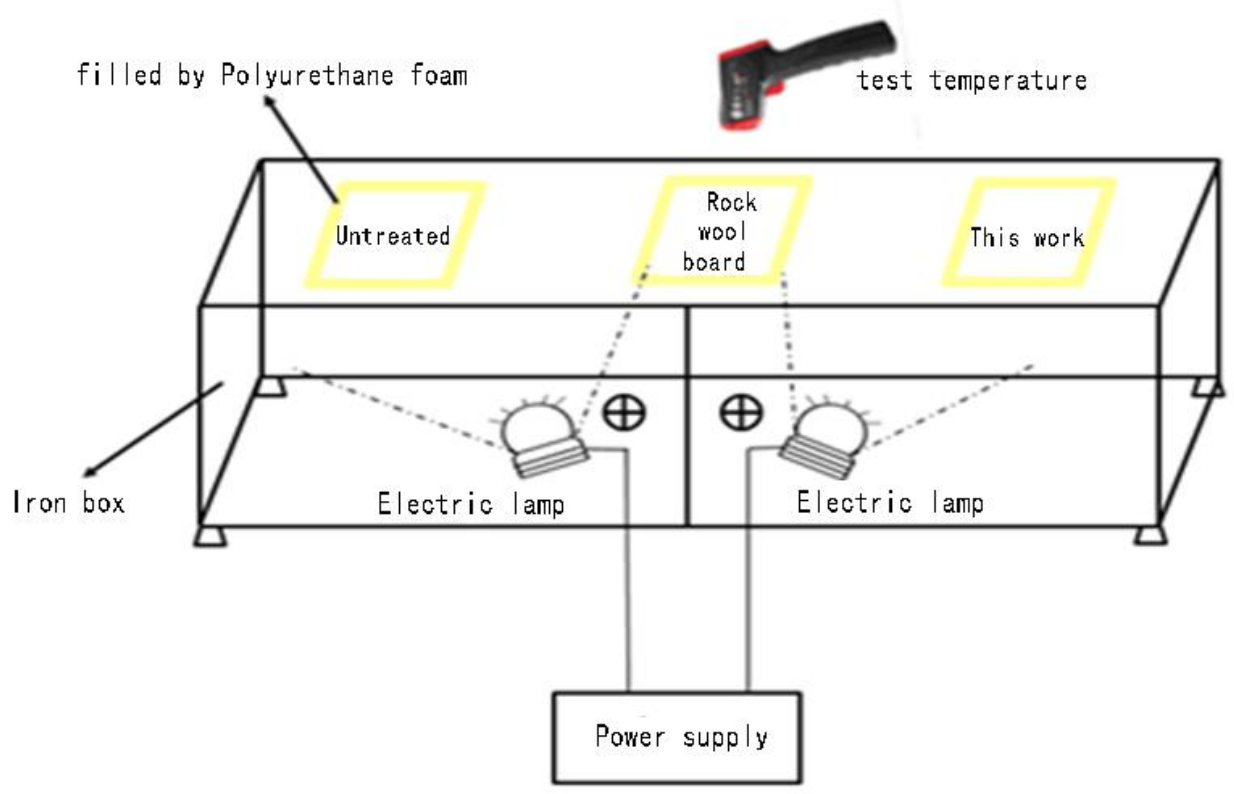

Figure 8 Insulation test device diagram

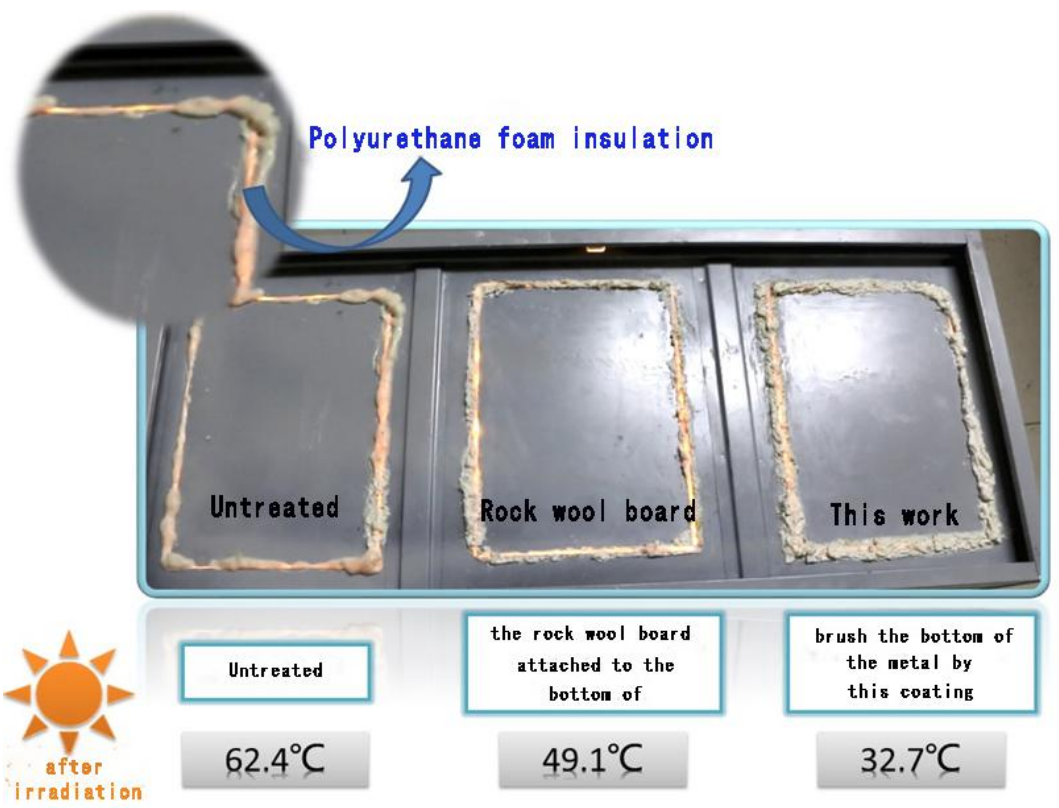

Figure 9 Test result

Partial temperature without treatment is $62.4{ }^{\circ} \mathrm{C}$, temperature sticking rock wool board is $49.1^{\circ} \mathrm{C}$, and temperature coated with new thermal reflective insulation coating is $32.7^{\circ} \mathrm{C}$. With the experimental results, it indicates that thermal reflective insulation coating can effectively block heat transfer. After this work is painted on indoor wall, the thermal barrier effect of the wall can be 
improved.

\section{Conclusion}

Under ultraviolet light, the titanium dioxide surface generates free radicals to cause polymerization of acrylamide monomers. Through the infrared spectrum, it can be seen that the absorption peak of the product increases gradually with the extension of the light time; it can be seen from the liquid chromatogram that the titanium dioxide causes the polymerization of acrylamide monomer; the polyacrylamide is coated on the surface of titanium dioxide by transmission electron microscope. By conducting absorbance test, it indicates the dispersion stability of titanium dioxide has been improved after modification; with reference to the literature method, the modified titanium dioxide is prepared into thermal reflective insulation coating, to test the thermal barrier effect. The test results show that the thermal insulation performance is much higher than that rock wool board and traditional insulating material. The method has a certain application value.

\section{References}

[1] Qingzhong Que. A Brief Discussion on Application of Architectural Reflective Insulation Coating in Construction Engineering[J]. Sichuan Building Materials, 2017, (05): 4-5. (in Chinese)

[2] Junshan Xiong, Qunying Wang, Sheng Cheng et al. Titanium Dioxide Modification and Its Application in Heat Reflection Coating[J]. New Building Materials, 2010, (05):85-88. (in Chinese) [3] Dandan Ye, Qingzhen Wen, Jinhua Zhu, et al. Research Progress of Heat Reflection Materials for Oil Tank at Home and Abroad[J]. Material Development and Application, 2017, 01:103-108. (in Chinese)

[4] Jianping Sun, Tao Wang, Xi Zhao, et al. Dispersion Technical Progress of Titanium Dioxide in Coating [J]. China Paint, 2013, (05):10-13 + 17. (in Chinese)

[5] Vincent Delobelle, Hervé Louche, Denis Favier.2014, Numerical study on the effect of the paint layer used for infrared thermography on heat source
es-timation[J]. Quantitative InfraRed Thermography Journal,11(2):73-77. (in Chinese)

[6] Rui Chen, Jungang Lv, Jimin Feng. 2015, Characterization of Paint by Fourier-Transform Infrared Spectroscopy, Raman Microscopy, and Scanning Electron Mi croscopy-Energy Dispersive X-ray Spectroscopy [J]. Analytical Letters, 48(9):98-103. (in Chinese)

[7] Guozhong Lu. Preparation of Architectural Reflection Insulating Mould Coatings [J]. China Paint, 2007, (09):37-40 + 42. (in Chinese)

[8] Bo Yuan. 2016. Research on preparation of heat insulation coatings and gradation optimization of pigments and fillers[D]. Southeast University. (in Chinese)

[9] Yu'ning Pang.2016. Preparation and study of silicone modified epoxy resin coating[D]. Shenyang University of Technology. (in Chinese)

[10] Luwei Shen.2016. Research on preparation of heat insulation coatings and gradation optimization of pigments and fillers[D]. Southeast University. (in Chinese) 